Research Article

\section{Dry eye syndrome: Therapeutic challenges and future trends}

\section{Irami Araújo-Neto ${ }^{1}$, Amália Cinhtia Meneses Rêgo² and Irami Araújo-Filho ${ }^{3 *}$}

${ }^{1}$ Undergraduate Student of Medicine, UnP - Potiguar University, Laureate International Universities, Natal, Brazil

${ }^{2}$ Postgraduate Program in Biotechnology at Potiguar University/ UnP - Laureate International Universities, Natal, Brazil. Ph.D. Health Science, Natal, RN, Brazil

${ }^{3}$ Postgraduate Program in Biotechnology, Potiguar University/ UnP - Laureate International Universities, Full Professor Department of Surgery, Federal University of Rio Grande do Norte. Full Professor, Department of Surgery, Potiguar University, Ph.D, Health Science, Natal, RN, Brazil

\section{Abstract}

Introduction: Intense Pulsed Light (IPL) is the therapeutic tool implemented in the treatment of dry eye, characterized by the emission of waves of 500-1200nm in the region of the upper and lower eyelids. Dry eye is a recurring complaint in ophthalmology offices and has an impact on the patient's quality of life. It results from dysfunction of the meibomian glands, duct obstruction, and quantitative and qualitative changes in glandular secretion. It is manifested by dryness and irritation, foreign body sensation, burning, tearing, and eye fatigue. The available treatments are short term palliative with unsatisfactory results.

Objective: To determine through a literature review, the benefit of using intense pulsed light for dry eye treatment. Methodology: We searched the Cochrane database, PubMed, Embase, Scopus, Web of Science, and Google Scholar (gray literature) using the keywords for an independent, blinded, peer-reviewed selection of articles to be met following pre-established criteria.

Results and discussion: Studies evaluated the use of IPL in patients with dry eye, despite the benefit found, focus on the objective and subjective assessment of the eye more frequently.

Conclusion: Evidence-based clinical guidelines are required for the use of intense pulsed light in the treatment of Meibomian Gland Dysfunction (MGD), as well as a device and algorithm for indications of therapeutic use.

\section{More Information}

*Address for Correspondence: Irami AraújoFilho, MD, Ph.D, Postgraduate Program in Biotechnology, Potiguar University, UnP - Laureate International Universities, Full Professor, Department of Surgery, Federal University of Rio Grande do Norte, Full Professor, Department of Surgery, Potiguar University, Ph.D, Health Science, Natal-RN, Brazil, Tel: +55 84 98876-0206; Email: irami.filho@uol.com.br

Submitted: 23 November 2019

Approved: 11 December 2019 Published: 12 December 2019

How to cite this article: Araújo-Neto I, Rêgo ACM, Araújo-Filho I. Dry eye syndrome: Therapeutic challenges and future trends. Int J Clin Exp Ophthalmol. 2019; 3: 030-034.

DOI: dx.doi.org/10.29328/journal.ijceo.1001023

ORCiD: orcid.org/0000-0003-2471-7447

Copyright: ( 2019 Araújo-Neto l, et al. This is an open access article distributed under the Creative Commons Attribution License, which permits unrestricted use, distribution, and reproduction in any medium, provided the original work is properly cited.

Keywords: Dry eye syndromes; Intense pulsed light therapy; Meibomian glands; Tarsal gland; Phototherapy; Eye diseases

D) Check for updates

\section{Introduction}

Dry Eye Syndrome (DES) is a multifactorial condition due to loss of tear film homeostasis. It has a high incidence. It is prevalent in $5 \%-50 \%$ of the world population and has a direct relationship with advancing age, female gender, and Asian ethnicity (60\%) [1,2]. It can be classified according to deficiency of aqueous tear production or excessive evaporation; beyond the two concomitant situations [2-4]. Historically, aqueous deficiency has represented for ophthalmology, the primary pathophysiological substrate in the genesis of the syndrome. However, new studies show that $80 \%$ of patients have evaporative or mixed-type dysfunction mechanisms $[1,5]$. Meibomian gland dysfunction (MGD) is the primary cause of evaporative dry eye and contributes to the development of the aqueous subtype. Glandular dysfunction generates tear film instability, resulting from hyperkeratinization and obstruction of external gland orifices with abnormal production of the lipid layer in qualitative and quantitative terms [4-6]. The meibomian glands (acinar-tarsal) are modified sebaceous follicles present in the upper and lower eyelids. These glands are distributed along each eyelid and secrete meibum, the lipid component of tears. In dysfunction, the organs became narrow, with atrophy of hyperkeratinized acini and increased the viscosity of the meibum, which reduces glandular flow and stimulates bacterial proliferation. Under these conditions, patients present increased tear evaporation with altered osmolarity, greater susceptibility to ocular surface inflammation, epithelial damage, and discomfort $[2,6]$. It is clinically manifested through dryness, irritation, 
foreign body sensation, burning, excessive tearing, redness, itching, fatigue, recurrent infections, and transient visual disturbances [4]. Some studies have compared the impact on the quality of life of patients with dry eye to moderate / severe angina or dialysis treatment [7]. Thus, the symptoms may represent a severe functional impairment to the individual with DES. Conventional therapies used for MGD have proven reduced efficacy in the moderate/severe stages of the disease. Traditional medicines include warm compresses, eyelid massage, and artificial tears. Other treatments involve the use of topical steroids, antibiotics, immunomodulatory agents, and fatty acids (omega-3) [1,8]. These therapies have shown a slight reduction in signs and symptoms of MGD and have been used for years to treat mild forms of the disease. However, they have adverse effects, high cost, limited accessibility, low levels of scientific evidence, need for continuous treatment for a better outcome, and potential induction of antimicrobial resistance [1-3]. For this reason, it is invested in the practice of dilation with an intraductal tube and continuous expression of the meibomian glands (EMG). EMG, although an invasive and painful procedure for the patient, has shown a long-term beneficial effect $[2,5]$. A recent alternative implemented in the treatment of MGD is the use of Intense Pulsed Light (IPL). It consists of the emission of visible, high-intensity polychromatic light at wavelengths of $500-1200 \mathrm{~nm}$, through equipment adapted with xenon flash lamp [7-9]. The emitted light is capable of producing perfectly calibrated and homogeneous light pulse sequences. Each pulse is released with distance, energy, and spectrum predetermined by the equipment to stimulate the meibomian glands and restore normal functioning $[1,8]$. The IPL is widely used in dermatology and has been incidentally incorporated into ophthalmology. Their discovery came from the observation of clinical improvement of DES in patients using IPL to treat periocular dermatological lesions. [5,8]. This study aimed to review the use of Intense Pulsed Light therapy to treat Dry Eye Syndrome. The benefits of conventional treatments and long-term IPL were compared. Prospective studies currently require significant samples to validate the real impact of this therapy [8-10].

\section{Material and Methods}

Cochrane, PubMed, Embase, Scopus, Web of Science, and Google Scholar databases (gray literature) using the keywords: dry eye syndromes, intense pulsed light therapy, meibomian glands, tarsal gland, phototherapy, eye diseases. Articles related to intense pulsed light treatment in dry eye syndrome resulting from meibomian gland dysfunction were selected. The filters involved studies of the last five years in humans published from July 2014 to July 2019. The Jadad and Newcastle-Ottawa scales were used to qualify and select articles on the subject. Study selection was independent, blinded, and peer-reviewed according to the inclusion criteria. Initially, 29 epidemiological studies were selected. Of these, eight studies that did not correspond to the research objective were discarded. After this stage, 21 studies were included in the present review.

\section{Results and Discussion}

\section{IPL therapy}

Intense Pulsed Light was proposed as a therapeutic option in 1989 by Morgan Gustavson, and his first model was presented in Stockholm the following year. It continued in development in subsequent years until the FDA regulated it in 1995 [3]. Since then, it has begun to be incorporated into dermatological practice for the treatment of rosacea, telangiectasia, acne, and other skin conditions. Its application improved in the following years, being proposed in 2002 possible benefits in ophthalmic diseases [3-5]. Ophthalmologist Rolando Toyos was the first to suggest the use of IPL for the treatment of MGD, from the observation of objective and subjective improvement of DES symptoms in patients with periocular rosacea undergoing Pulsed Light therapy [8-10]. However, it has been proven that the effectiveness of treatment in the evaporative dry eye is independent of the presence of a previous dermatological lesion. After that, therapy has undergone successive studies and adaptations in the field of ophthalmology to improve its applicability [5]. In 2007, the first equipment designed explicitly for ophthalmology was developed, ensuring a more accurate and useful standard in treating MGD. The device uses a broad-spectrum polychromatic light source with a wavelength of 500-1.200 nm, selectively absorbed by various chromophores (hemoglobin, melanin, and water) in the skin. This light is filtered by the source equipment to allow selective wavelength emission [9].

\section{Mechanisms of action}

The mechanisms of action of IPL in the treatment of evaporativedry eye arestill being elucidated, with nine different hypotheses so far: abnormal blood vessel thrombosis, meibum heating, and liquefaction, epithelial turnover reduction, photomodulation, fibroblast activation, Demodex eradication. , modulation and secretion of pro and anti-inflammatory molecules, suppression of matrix metalloproteases, and release of reactive oxidative species $[1,3]$. The most widely accepted hypothesis deals with thrombosis of abnormal blood vessels by transforming light absorbed by hemoglobin into heat, reducing the reserve of inflammatory mediators in the eyelid and meibomian glands [9-11]. The temperature of the eyelid physically influences the meibum. High temperatures increase its distribution in the cornea as a result of reduced viscosity, facilitating the clearance of glands and glandular expression [10-12]. Thus, the symptomatic improvement of dry eye is due to the restoration of the meibum during blinking. Epithelial turnover is a risk factor for occlusion of the meibomian glands by the accumulation of debris on the eyelid margin. Consequently, by reducing this mechanism, IPL acts as a protective factor against obstruction [3,7-9]. The lack of collagen fibers causes a reduction in meibomian pumping, leading to a decrease in TRFL (tear film rupture time). From photomodulation, there is stimulation of fibroblast proliferation and increased collagen concentration in the 
skin, increasing the pressure required on the eyelid margin and normalizing glandular drainage [5-7,13]. Demodex is a type of ectoparasite present in the sebaceous and meibomian glands. Its proliferation causes the increase of bacteria in the eyelid region, among them, Bacillus oleronius [6-8]. This bacterium elicits a chronic inflammatory response and acts locally through the production of toxic substances that modify the tear lipid layer $[7,14]$. Therefore, eradication through coagulation and necrosis inhibits the modulation mechanism of blepharitis and, consequently, MGD. IPL acts in three ways of regulating the inflammatory process. The proposed mechanism comprises the regulation of anti-inflammatory cytokines and the regulation of proinflammatory cytokines $[3,15,16]$. Other types of proteins involved in dry eye pathogenesis are metalloproteases (MMPs). These enzymes act on extracellular matrix remodeling and are directly and indirectly affected by IPL [3]. IPL attenuates Reactive Oxidative Species (ROS) levels, reducing oxidative stress and inflammation caused by neutrophils and other inflammatory cells [3]. Pulsed light makes changes in many ways. The emitted photons induce a photochemical cascade, increased ATP, and intracellular calcium. This calcium outbreak activates cellular responses in fibroblasts by stimulating cell proliferation, collagen synthesis, and improved blood flow. Macrophages are enabled, and keratinocytes increase the secretion of pro or anti-inflammatory cytokines, depending on the context $[3,15]$. Mejía, et al. stated that this is the primary pathophysiological mechanism that can explain the photochemical stimulation of the meibomian glands. The author came to this conclusion from comparative studies that showed little relevance or were limited to the adjuvant effect on treatment [1].

\section{Intense pulsed light therapy}

IPL grading should be made according to the patient's skin type using the Fitzpatrick phototype scale, which ranges from I to VI. Treatment eligibility is contraindicated at levels $\mathrm{V}$ and VI. The light intensity shall be inversely proportional to the graduation on the prototype scale. Patients with higher melanin concentration in the skin require lower energy adjustments to avoid light hypopigmentation [4,5]. Another factor that should be considered is the severity of the disease, and for this, the light source is adjusted to power between $8-20 \mathrm{~J} / \mathrm{cm}^{2}$, with indication proportional to MGD. Aspects of tolerance and comfort should also be considered $[4,8]$.

\section{IPL application steps:}

- Place the patient in a comfortable position in the office, sitting or lying down

- Turn on the device, select the specific treatment mode and set the power level

- Confirm the parameters set in the equipment

- Put the goggles on yourself and the patient during the procedure
- Apply the ultrasound gel to the patient's face, wrapping the area between the tragus, including the nose. This layer must be at least $1 \mathrm{~cm}$ thick

- Perform a IPL pulse cycle

- After this, the patient receives a new gel layer, and one more IPL pass is performed

- The gel layer should be carefully peeled off and a drop of $1 \%$ procaine for later expression of the glands

- The patient is instructed to look up, and the feeling between the finger and the inner eye is performed for the $30 \mathrm{~s}$

- The procedure may be repeated on the other eye if necessary

- For upper eyelid expression, the patient should look down, and the same technique is performed. In combination with treatment, a drop of topical corticosteroid or non-steroidal anti-inflammatory is prescribed if gland expression is required

Most patients undergo a series of four sessions, with an average periodicity of 3-6 weeks and maintenance between 4-12 months. However, the studies are conflicting and do not follow an established pattern [4,14-16].

\section{Treatment effectiveness}

Studies are consonant regarding the benefits of IPL for the treatment of MGD. However, there are tests with discreet conflicting results that require adequacy. For this reason, the purpose of this topic is to highlight the positive and negative aspects that were evaluated [11-13]. Craig, et al. reported a study of 28 patients with dry eye resulting from MGD. They found an improvement in tear film rupture time (TFRT) and in the eyepiece lipid layer. This benefit was assessed from subjective symptom scores, SPEED form [17]. Toyos, et al. analyzed 91 patients observed an improvement in TFRT and general symptoms in $87 \%-93 \%$ of cases [8]. There was an improvement in TFRT in $87 \%$ of patients undergoing seven therapeutic sessions and four maintenance sessions with IPL. Besides, subjective symptomatic improvement was observed in $93 \%$ of DES patients undergoing therapy $[8,10,11,21]$. In the study by Vora, et al. 37 DES patients were treated with IPL and evaluated by the Ocular Surface Disease Index (OSDI). Decreased clinical signs of MGD, eyelid edema and improved meibum quality were found. Also, increased TFRT and better lipid layer distribution have been proven $[10,11]$. TFRT increased throughout IPL application, varying by $4.9 \mathrm{~s}$, due to the qualitative improvement of the meibum and ease of expression of the glands [11.12]. Another viable alternative is the combination of IPL and meibomian gland expression (MGE). Rong, et al. evaluated this association and demonstrated prolonged relief of signs of DES symptoms 
for at least six months [9]. Vegunta, et al. showed through a retrospective study with 81 patients undergoing combination therapy, clinical improvement in $89 \%$ of patients with DES, and $77 \%$ of patients with MGD [2]. Arita, et al. compared MGE therapy with the MGE/IPL combination in a study of 45 patients. Combination therapy was superior in improving the lipid component of the tear film compared with MGE alone. MGE/IPL treatment also showed good clinical evolution after the application of objective and subjective DES tests [13]. However, Craig and Jiang, et al., when doing treatment without MGE, also reported significant improvement in the qualitative component of the lipid layer, TFRT, and symptomatic scores [17]. Therefore, further studies with more significant sample numbers are needed to evaluate isolated IPL and compared it with MGE-associated IPL [12]. Comparing the success rate obtained in the eyelids, we found greater efficacy of treatment in the lower eyelids. This is due to the higher accumulation of inflammatory factors in this region, and since IPL acts to reduce the buildup of inflammatory molecules, this favorable factor was observed. [7-9]. To quantify this benefit, patients were followed for $1,3,6$, and 9 months, with the best results found in the lower eyelids, achieving success rates of $377.9 \%$, $339.7 \%, 278.4 \%$, and $147 \%$, respectively. Regarding the upper eyelids, the most significant number found was $81.2 \%$ improvement [8-11]. A study developed by Vora, et al. showed prolonged symptomatic relief in patients undergoing therapy, demonstrating that maintenance therapy is required only every 6-12 months. Rong, et al. It also indicated maintenance application from 6 months, reiterating the association with MGE $[10,11]$. This maintenance period is uncertain as no studies addressing all improvements in one year were found. As an example, the TFRT impact assessment showed no significant improvement over the full 6-12 months after IPL onset $[10,11,14]$. Seo, et al. analyzed patients with rosacea and MGD and revealed improvements in eye symptoms from three weeks until the examination in the twelfth month. Among them, the vascularization of the eyelid margin, expressiveness of the meibum, and the quality of this fluid persisted until the end of follow-up [14]. However, the first prospective analysis article to quantify clinical improvements in signs and symptoms was that of Albietz, et al. in 2017. While clinical signs improved after three sessions, symptoms only improved after six weeks of final treatment [12]. Regardless, $85 \%$ of participants had improved subjective and objective measures of symptomatology. Vegunta, Patel, and Shen had the same incidence of success, with $89 \%$ of cases improving on the SPEED test. This test is indicated to correlate the evaporative dry eye parameters $[2,12]$. Another parameter evaluated by researchers Craig, Chen, and Turnbull was osmolarity and tear evaporation rate, but no changes were found. Meanwhile, Toyos reported a minimal reduction in tear osmolarity after IPL [8]. Nevertheless, this data is clinically irrelevant, as it does not interfere with symptoms [12,17]. Guilloto, et al. demonstrated the relationship between IPL treatment refractoriness and previous eye surgery. A total of 72 eyes examined, those without last surgery or operated with phacoemulsification and photorefractive keratectomy (PRK), the therapy was useful. On the other hand, in patients undergoing refractive surgery with Lasik Femtosegundo ${ }^{\circledR}$ (LFS) or the use of mitomycin (MM), no improvement in DES was observed. This is due to the laser-induced corneal stromal ablation, showing that the DES of these patients is related to the corneal response, not to MGD [15]. In this sense, the Fitzpatrick scale is a patient selector component for treatment. Darker skins are more likely to endothelial damage due to the higher amount of melanin. Li, et al. analyzed the use of IPL in patients with MGD and Fitzpatrick III / IV scale. The group that received pulses with $590 \mathrm{~nm}$ of wavelength and $14 \mathrm{~mJ} / \mathrm{cm}^{2}$ of energy obtained a better therapeutic response, is considered safe and effective for patients with this skin tone [16]. Regarding the antibacterial effects of IPL, studies suggest the relationship of light with a reduction in bacterial colony count. Still, it is unlikely to have a significant effect when goggles protect eyelid margins. Besides, this region has a higher prevalence of negative $S$. aureus and S. coagulase, poor in porphyrins, and therefore does not respond to IPL therapy [12]. The time to realize significant benefits begins within hours of treatment. In the study by Karaca, et al. of the 26 patients, 20 reported symptomatic relief on the same day and the remainder after the second session. Complete treatment success is assumed to depend on the completion of three sessions; however, another cycle may be required. During the follow-up of these patients for six months, none of them had a new session [17]. To further elucidate favorable results from the mechanism of the genesis of MGD, much research is underway. Recently, a randomized, double-blind study demonstrated a reduction in cytokines (IL6 and IL17) and prostaglandin E2 with the use of IPL. Dry eye cytokine analysis caused by MGD showed an increase in CX3CL1, IL1Ra, IL-6, IL-8 / CXCL8, and EGF [12-14]. These markers should be further investigated for understanding the antiinflammatory mechanism and posttreatment measurement and a possible relationship with IPL failure [18-20].

\section{Treatment iimitations}

Eye reactions to treatment have been observed due to inadequate eye protection: anterior uveitis, iris atrophy, pupillary defects, and periorbital pain. Other effects are discomfort and erythema that improve over time [10]. Study limitations involve loss of follow-up due to several reasons, which affects sample representativeness. There are other difficulties regarding the high number of re-evaluation visits and the impossibility of submitting patients who have the same severity of MGD to different treatments and without potential benefit $[12,21]$.

\section{Conclusion}

In conclusion, IPL has beneficial effects in the treatment of DES, but there is much to discover about replication today. Prospective randomized studies with a representative number 
of patients are imperative to confirm the impact of IPL on this highly prevalent disease that is difficult to confirm diagnose in ophthalmic practice. Advances in the treatment of DES and the use of IPL have been significant; however, ongoing studies are needed in search of new therapeutic methods that promote better clinical outcomes in the treatment of this condition.

\section{Acknowledgment}

The authors thank the Ophthalmologist, Prof. Dr. Franciso Irochima Pinheiro, for his contribution and discussion of a clinical case of significant scientific relevance and the supervision of this review, acting as an expert consultant on the bibliographic survey, analysis, and scientific advice. We also thank all the study components for their dedication and effort to build a scientifically validated quality review.

\section{References}

1. Mejía LF, Gil JC, Jaramillo M. Intense pulsed light therapy: A promising complementary treatment for dry eye disease. Arch Soc Esp Oftalmol. 2019; 94: 331-336.

PubMed: https://www.ncbi.nlm.nih.gov/pubmed/31079987

2. Vegunta S, Patel D, Shen JF. Combination Therapy of Intense Pulsed Light Therapy and Meibomian Gland Expression (IPL/MGX) Can Improve Dry Eye Symptoms and Meibomian Gland Function in Patients With Refractory Dry Eye: A Retrospective Analysis. Cornea. 2016; 35: 318-322.

PubMed: https://www.ncbi.nlm.nih.gov/pubmed/26785301

3. Dell SJ. Intense pulsed light for evaporative dry eye disease. Clin Ophthalmol. 2017; 20; 11: 1167-1173.

PubMed: https://www.ncbi.nlm.nih.gov/pubmed/28790801

4. Vigo L, Giannaccare G, Sebastiani S, Pellegrini M, Carones F. Intense Pulsed Light for the Treatment of Dry Eye Owing to Meibomian Gland Dysfunction. J Vis Exp. 2019.

PubMed: https://www.ncbi.nlm.nih.gov/pubmed/30985743

5. Dell SJ, Gaster RN, Barbarino SC, Cunningham DN. Prospective evaluation of intense pulsed light and meibomian gland expression efficacy on relieving signs and symptoms of dry eye disease due to meibomian gland dysfunction. Clin Ophthalmol. 2017; 11: 817-827. PubMed: https://www.ncbi.nlm.nih.gov/pubmed/28496300

6. Rong B, Tang Y, Tu P, Liu R, Qiao J, et al. Intense Pulsed Light Applied Directly on Eyelids Combined with Meibomian Gland Expression to Treat Meibomian Gland Dysfunction. Photomed Laser Surg. 2018; 36 : 326-332.

PubMed: https://www.ncbi.nlm.nih.gov/pubmed/29688838

7. Gupta PK, Vora GK, Matossian C, Kim M, Stinnett S. Outcomes of intense pulsed light therapy for treatment of evaporative dry eye disease. Can J Ophthalmol. 2016; 51: 249-253.

PubMed: https://www.ncbi.nlm.nih.gov/pubmed/27521662

8. Toyos R, McGill W, Briscoe D. Intense pulsed light treatment for dry eye disease due to meibomian gland dysfunction; a 3-year retrospective study. Photomed Laser Surg. 2015; 33: 41-46.

PubMed: https://www.ncbi.nlm.nih.gov/pubmed/25594770
9. Rong B, Tang Y, Liu R, Tu P, Qiao J, et al. Long-Term Effects of Intense Pulsed Light Combined with Meibomian Gland Expression in the Treatment of Meibomian Gland Dysfunction. Photomed Laser Surg. 2018; 36: 562-567.

PubMed: https://www.ncbi.nlm.nih.gov/pubmed/30251914

10. Vora GK, Gupta PK. Intense pulsed light therapy for the treatment of evaporative dry eye disease. Curr Opin Ophthalmol. 2015; 26: 314-318. PubMed: https://www.ncbi.nlm.nih.gov/pubmed/26058031

11. Mandal P, Khan MI, Shah S. Drugs - Do we need them? Applications of non- pharmaceutical therapy in anterior eye disease: A review. Cont Lens Anterior Eye. 2017; 40: 360-366.

PubMed: https://www.ncbi.nlm.nih.gov/pubmed/28919243

12. Albietz JM, Schmid KL. Intense pulsed light treatment and meibomian gland expression for moderate to advanced meibomian gland dysfunction. Clin Exp Optom. 2018; 101: 23-33.

PubMed: https://www.ncbi.nlm.nih.gov/pubmed/28585267

13. Arita R, Fukuoka S, Morishige N. Therapeutic efficacy of intense pulsed light in patients with refractory meibomian gland dysfunction. Ocul Surf. 2019; 17: 104-110.

PubMed: https://www.ncbi.nlm.nih.gov/pubmed/30445177

14. Seo KY, Kang SM, Ha DY, Chin HS, Jung JW. Long-term effects of intense pulsed light treatment on the ocular surface in patients with rosacea-associated meibomian gland dysfunction. Cont Lens Anterior Eye. 2018; 41: 430-435.

PubMed: https://www.ncbi.nlm.nih.gov/pubmed/29958778

15. Guilloto SC, García JLM, Colmenero ER. Effect of pulsed laser light in patients with dry eye syndrome. Arch Soc Esp Oftalmol. 2017; 92: 509-515. PubMed: https://www.ncbi.nlm.nih.gov/pubmed/28256362

16. Li D, Lin S, Cheng B. Intense Pulsed Light Treatment for Meibomian Gland Dysfunction in Skin Types III/IV. Photobiomodulation, Photomedicine, and Laser Surgery. 2019; 37: 70-76. PubMed: https://www.ncbi.nlm.nih.gov/pubmed/31050931

17. Karaca EE, Evren Kemer Ö, Özek D. Intense regulated pulse light for the meibomian gland dysfunction. Eur J Ophthalmol. 2018. PubMed: https://www.ncbi.nlm.nih.gov/pubmed/30514116

18. Al-Saedi Z, Zimmerman A, Bachu RD, Dey S, Shah Z, et al. Dry Eye Disease: Present Challenges in the Management and Future Trends. Curr Pharm Des. 2016; 22: 4470-4490.

PubMed: https://www.ncbi.nlm.nih.gov/pubmed/27296759

19. Liu R, Rong B, Tu P, Tang Y, Song W, et al. Analysis of Cytokine Levels in Tears and Clinical Correlations After Intense Pulsed Light Treating Meibomian Gland Dysfunction. American Journal of Ophthalmology. 2017; 183: 81-90.

PubMed: https://www.ncbi.nlm.nih.gov/pubmed/28887117

20. Song WJ, Yan XM. Research progress of intense pulsed light treatment on meibomian gland dysfunction and relevant dry eye diseases. Europe PMC. 2018; 54:140-143.

PubMed: https://www.ncbi.nlm.nih.gov/pubmed/29429299

21. Craig JP, Chen YH, Turnbull PRK. Prospective Trial of Intense Pulsed Light for the Treatment of Meibomian Gland Dysfunction. Investigative Opthalmology \& Visual Science. 2015; 56: 1-14.

PubMed: https://www.ncbi.nlm.nih.gov/pubmed/25678687 